\title{
A Property of the Weak Subalgebra Lattice for Algebras with Some Non-Equalities
}

KONRAD PIÓRO

Institute of Mathematics, University of Warsaw, ul. Banacha 2, 02-097 Warsaw, Poland

e-mail : kpioro@mimuw.edu.pl

Abstract. Let $\mathbf{A}$ be a locally finite total algebra of finite type such that $k^{\mathbf{A}}\left(a_{1}, \ldots, a_{n}\right) \neq$ $a_{i}$ for every operation $k^{\mathbf{A}}$, elements $a_{1}, \ldots, a_{n}$ and $1 \leq i \leq n$. We show that the weak subalgebra lattice of $\mathbf{A}$ uniquely determines its (strong) subalgebra lattice. More precisely, for any algebra $\mathbf{B}$ of the same finite type, if the weak subalgebra lattices of $\mathbf{A}$ and $\mathbf{B}$ are isomorphic, then their subalgebra lattices are also isomorphic. Moreover, $\mathbf{B}$ is also total and locally finite.

\section{Introduction}

A type of algebra is a pair $\langle K, \kappa\rangle$, where $K$ is a set of operation symbols and $\kappa$ is an arity function from $K$ into the set of all non-negative integers $\mathbb{N}$. Note that 0 -ary operation symbols $k$ (i.e., $\kappa(k)=0$ ) are just symbols of constants. $\langle K, \kappa\rangle$ is a finite type if $K$ is finite.

A partial algebra of (algebraic) type $\langle K, \kappa\rangle$ is a pair $\mathbf{A}=\left\langle A,\left(k^{\mathbf{A}}\right)_{k \in K}\right\rangle$, where $A$ is a set of elements of $\mathbf{A}$ and for any $k \in K, k^{\mathbf{A}}: A^{\kappa(k)} \longrightarrow A$ is a $\kappa(k)$-ary partial operation of $\mathbf{A}$, i.e., $k^{\mathbf{A}}$ is only defined on a subset of $A^{\kappa(k)}$ (see e.g., [3] and [5]). Taking total operations instead of partial ones we obtain the well-known definition of a (total) algebra. More formally, a $\kappa(k)$-ary operation $k^{\mathbf{A}}: A^{\kappa(k)} \longrightarrow A$ is total, if $k^{\mathbf{A}}\left(a_{1}, \ldots, a_{\kappa(k)}\right)$ is defined for each $\left(a_{1}, \ldots, a_{\kappa(k)}\right) \in A^{\kappa(k)}$ (see e.g., [7] or [9]). Of course, each total algebra is partial.

Recall also (see e.g., [9]) that a lattice $\mathcal{L}=\langle L, \leq\rangle$ is complete, if each subset $S \subseteq L$ has an infimum $\bigwedge S$ and a supremum $\bigvee S$. A complete lattice $\mathcal{L}$ is algebraic, if each of its elements is the supremum of some set of compact elements. An element $c \in L$ is compact, if for each set $S \subseteq L, c \leq \bigvee S$ implies $c \leq \bigvee S_{0}$ for some finite subset $S_{0} \subseteq S$.

The concept of subalgebra can be transported onto the partial case without changes. Formally, we say that a subset $B$ of elements of a (partial) algebra $\mathbf{A}=$ $\left\langle A,\left(k^{\mathbf{A}}\right)_{k \in K}\right\rangle$ is closed under operations, if for any operation $k^{\mathbf{A}}$ and each sequence

Received July 9, 2008; revised December 16, 2008; accepted October 9, 2009.

2000 Mathematics Subject Classification: 05C65, 05C99, 08A30 (05C90, 08A55).

Key words and phrases: hypergraph, strong and weak subalgebras, subalgebra lattices, partial algebra. 
$\left(b_{1}, \ldots, b_{\kappa(k)}\right) \in B^{\kappa(k)}$, if $k^{\mathbf{A}}\left(b_{1}, \ldots, b_{\kappa(k)}\right)$ is defined, then $k^{\mathbf{A}}\left(b_{1}, \ldots, b_{\kappa(k)}\right) \in B$. This set $B$ together with operations of $\mathbf{A}$ restricted to $B$ forms a subalgebra of A. Such subalgebras will sometimes be called strong here to distinguish them from other kinds of partial subalgebras which may be defined in the partial case (see e.g., [3] or [5]).

It is a classical fact, true also in the partial case, that the family of all strong subalgebras of a (partial) algebra $\mathbf{A}$ with subalgebra inclusion $\leq_{s}$, forms a lattice $\mathcal{S}_{s}(\mathbf{A})=\left\langle S_{s}(\mathbf{A}), \leq_{s}\right\rangle$. This lattice is complete and algebraic (see e.g., [9]).

In particular, for any subset $W \subseteq A$ of elements of $\mathbf{A}$, there is the least strong subalgebra containing $W$, which will be denoted by $\langle W\rangle_{\mathbf{A}}$.

Recall that a partial algebra $\mathbf{A}$ is locally finite, if for each finite set $W$ of elements of $\mathbf{A},\langle W\rangle_{\mathbf{A}}$ is a finite subalgebra.

The lattice of subalgebras and connections between (total) algebras and their subalgebra lattices are an important part of universal algebra and are also important for classical algebras. For instance, there are many results describing subalgebra lattices for algebras belonging to a given variety or a given type (see e.g., [9] or [10]), some such questions are still open. Some authors investigate algebras or varieties of algebras with special subalgebra lattices, e.g., distributive, modular, etc. (see e.g., [8], [15], [16]). Note also that some part of group theory investigates connections between groups and their subgroup lattices (see [14]). For example recall the classical beautiful result due to Ore that a group has a distributive subgroup lattice if and only if it is locally cyclic (i.e., each finitely generated subgroup is cyclic).

The theory of partial algebras provides additional tools for such investigations. Besides the strong subalgebra, at least three different structures may be considered in this case (see e.g., [3] or [5]). Here we consider only the concept of weak subalgebra (it seems that it is the most important type of partial subalgebras) and the lattice of weak subalgebras. This lattice alone, and also together with the strong subalgebra lattice may yield some interesting information on partial and total algebras.

Let $\mathbf{A}=\left\langle A,\left(k^{\mathbf{A}}\right)_{k \in K}\right\rangle$ and $\mathbf{B}=\left\langle B,\left(k^{\mathbf{B}}\right)_{k \in K}\right\rangle$ be partial algebras of the same type $\langle K, \kappa\rangle$. Recall that $\mathbf{B}$ is a weak subalgebra of $\mathbf{A}$ (which is denoted by $\mathbf{B} \leq_{w}$ $\mathbf{A})$, if $B \subseteq A$ and for each $k \in K$ and any sequence $\left(b_{1}, \ldots, b_{\kappa(k)}\right) \in B^{\kappa(k)}$, if $k^{\mathbf{B}}\left(b_{1}, \ldots, b_{\kappa(k)}\right)$ is defined (in $\left.\mathbf{B}\right)$, then $k^{\mathbf{A}}\left(b_{1}, \ldots, b_{\kappa(k)}\right)$ is also defined (in $\mathbf{A}$ ) and $k^{\mathbf{B}}\left(b_{1}, \ldots, b_{\kappa(k)}\right)=k^{\mathbf{A}}\left(b_{1}, \ldots, b_{\kappa(k)}\right)$. Equivalently, we can say that $B \subseteq A$ and $k^{\mathbf{B}} \subseteq k^{\mathbf{A}}$ for any $k \in K$.

The family of all weak subalgebras of a partial algebra $\mathbf{A}$ with weak subalgebra inclusion $\leq_{w}$, also forms a lattice $\mathcal{S}_{w}(\mathbf{A})=\left\langle S_{w}(\mathbf{A}), \leq_{w}\right\rangle$ which is complete and algebraic. A complete characterization of the weak subalgebra lattice is given in $[1]$.

An interesting property of the weak subalgebra lattice is proved in [11]. More precisely, for any locally finite total unary algebra of finite type, its weak subalgebra lattice uniquely determines its strong subalgebra lattice. In the present paper we generalize this result for arbitrary locally finite total algebras $\mathbf{A}=\left\langle A,\left(k^{\mathbf{A}}\right)_{k \in K}\right\rangle$ of finite type $\langle K, \kappa\rangle$ such that $k^{\mathbf{A}}\left(a_{1}, \ldots, a_{\kappa(k)}\right) \neq a_{i}$ for each $k \in K$, any $a_{1}, \ldots, a_{\kappa(k)} \in A$ and $1 \leq i \leq \kappa(k)$. 


\section{Basic facts}

Papers [12] and [13] give some connections between algebras and hypergraphs, which will be useful in our investigation. More precisely, in this section we translate our algebraic problem into a hypergraph one, which will be solved in the next section. But first we recall some facts from these two papers.

Since we use hypergraphs to represent algebras, vertex and hyperedge sets may have arbitrary cardinality, and also multiple hyperedges and isolated vertices are admitted. Therefore we will use a slightly more formal definition than usual (see e.g., [4]).

An (undirected) hypergraph $\mathbf{H}$ is represented by a triple $\left\langle V^{\mathbf{H}}, E^{\mathbf{H}}, I^{\mathbf{H}}\right\rangle$, where $V^{\mathbf{H}}$ is the vertex set of $\mathbf{H}, E^{\mathbf{H}}$ is the hyperedge set of $\mathbf{H}$ and $I^{\mathbf{H}}$ is the incidence function of $\mathbf{H}$ i.e., $I^{\mathbf{H}}$ is a function from $E^{\mathbf{H}}$ into the set of all non-empty and finite subsets of $V^{\mathbf{H}}$ (for any $e \in E^{\mathbf{H}}, I^{\mathbf{H}}(e)$ is the set of all the endpoints of $e$ ).

We also need the concept of directed hypergraph, which is a simple generalization of a directed graph.

Definition 2.1. A dihypergraph (directed hypergraph) D is a triple $\left\langle V^{\mathbf{D}}, E^{\mathbf{D}}, I^{\mathbf{D}}\right\rangle$ such that $V^{\mathbf{D}}$ is the vertex set of $\mathbf{D}, E^{\mathbf{D}}$ is the hyperedge set of $\mathbf{D}$ and $I^{\mathbf{D}}=\left\langle I_{1}^{\mathbf{D}}, I_{2}^{\mathbf{D}}\right\rangle$ is the incidence function, i.e., $I_{1}^{\mathrm{D}}$ is a function from $E^{\mathrm{D}}$ into the family of all finite (also empty) subsets of $V^{\mathbf{D}}$ and $I_{2}^{\mathrm{D}}$ is a function from $E^{\mathbf{D}}$ into $V^{\mathrm{D}}$. For any hyperedge $e \in E^{\mathbf{H}}, I_{1}^{\mathrm{D}}(e)$ is the initial set of $e$ and $I_{2}^{\mathrm{D}}(e)$ is the final vertex of $e$.

Let $\mathbf{D}$ be a dihypergraph and $e \in E^{\mathbf{D}}$ its hyperedge. Then $e$ is said to be $a$ hyperloop, if its final vertex belongs to its initial set (i.e., $I_{2}^{\mathrm{D}}(e) \in I_{1}^{\mathrm{D}}(e)$ ), otherwise $e$ is regular. The set of all regular hyperedges is denoted by $E_{\text {reg }}^{\mathrm{D}}$, and the set of all hyperloops by $E_{l o}^{\mathrm{D}}$.

Further, we say that $e$ is $a k$-edge, if its initial set $I_{1}^{\mathrm{D}}(e)$ has exactly $k$ vertices (i.e., $\left.\left|I_{1}^{\mathrm{D}}(e)\right|=k\right)$. A hyperloop that is a $k$-edge is a $k$-loop. $E^{\mathbf{D}}(k)$ denotes the set of all the $k$-edges of $\mathbf{D}$.

By our definition, dihypergraphs may contain 0-edges, i.e., hyperedges $e$ such that $I_{1}^{\mathrm{D}}(e)=\emptyset$. Observe that 0 -edges can be identified, similarly as for algebras, with their final vertices, and therefore they are sometimes called constants, too.

For any dihypergraph $\mathbf{D}$, let $\mathbf{D}^{*}=\left\langle V^{\mathbf{D}^{*}}, E^{\mathbf{D}^{*}}, I^{\mathbf{D}^{*}}\right\rangle$ be a hypergraph obtained from $\mathbf{D}$ by omitting the orientation of all the hyperedges, i.e.,

$$
V^{\mathbf{D}^{*}}=V^{\mathbf{D}}, E^{\mathbf{D}^{*}}=E^{\mathbf{D}} \text { and } I^{\mathbf{D}^{*}}(e)=I_{1}^{\mathbf{D}}(e) \cup\left\{I_{2}^{\mathbf{D}}(e)\right\} \text { for each } e \in E^{\mathbf{D}} \text {. }
$$

With any partial algebra $\mathbf{A}=\left\langle A,\left(k^{\mathbf{A}}\right)_{k \in K}\right\rangle$ we can associate a dihypergraph $\mathbf{D}(\mathbf{A})$. Recall (see [12]) that $A$ is the vertex set of $\mathbf{D}(\mathbf{A})$, i.e.,

$$
V^{\mathbf{D}(\mathbf{A})}=A
$$

and hyperedges of $\mathbf{D}(\mathbf{A})$ are directed triples $\left\langle V_{\mathbf{a}}, k, k^{\mathbf{A}}(\mathbf{a})\right\rangle$ such that $k^{\mathbf{A}}$ is a $\kappa(k)$-ary operation of $\mathbf{A}, \mathbf{a}=\left(a_{1}, \ldots, a_{\kappa(k)}\right) \in A^{\kappa(k)}$ is a $\kappa(k)$-element sequence, 
$V_{\mathbf{a}}=\left\{a_{1}, \ldots, a_{\kappa(k)}\right\}$ is the set of all the pairwise different elements of $\mathbf{a}$ and the partial operation $k^{\mathbf{A}}$ is defined on a, i.e.,

$$
\begin{aligned}
E^{\mathbf{D}(\mathbf{A})}= & \left\{\left\langle V_{\mathbf{a}}, k, k^{\mathbf{A}}(\mathbf{a})\right\rangle: k \in K, \mathbf{a}=\left(a_{1}, \ldots, a_{\kappa(k)}\right) \in A^{\kappa(k)},\right. \\
& \left.V_{\mathbf{a}}=\left\{a_{1}, \ldots, a_{\kappa(k)}\right\}, k^{\mathbf{A}} \text { is defined on } \mathbf{a}\right\} .
\end{aligned}
$$

For any hyperedge $\left\langle V_{\mathbf{a}}, k, k^{\mathbf{A}}(\mathbf{a})\right\rangle, V_{\mathbf{a}}$ is the initial set of $e$ and $k^{\mathbf{A}}(\mathbf{a})$ is the final vertex of $e$, i.e.,

$$
I_{1}^{\mathbf{D}(\mathbf{A})}\left(\left\langle V_{\mathbf{a}}, k, k^{\mathbf{A}}(\mathbf{a})\right\rangle\right)=V_{\mathbf{a}} \text { and } I_{2}^{\mathbf{D}(\mathbf{A})}\left(\left\langle V_{\mathbf{a}}, k, k^{\mathbf{A}}(\mathbf{a})\right\rangle\right)=k^{\mathbf{A}}(\mathbf{a}) .
$$

Note that if $c^{\mathbf{A}}$ is a constant defined in $\mathbf{A}$, then it is represented by a 0 -edge of the form $\left\langle\emptyset, c, c^{\mathbf{A}}\right\rangle$, where $c \in K$ is the constant symbol corresponding to $c^{\mathbf{A}}$. Note also that $\mathbf{D}(\mathbf{A})$ can be considered as a dihypergraph labelled by the set $K$.

We can also associate with $\mathbf{A}$ the (undirected) hypergraph $\mathbf{D}(\mathbf{A})^{*}$.

The following result immediately follows from the above definitions.

Lemma 2.2 Let $\mathbf{A}$ be a partial algebra of type $\langle K, \kappa\rangle$. The following conditions are equivalent:

(a) $\mathbf{D}(\mathbf{A})$ has no hyperloops,

(b) for any $k \in K$, elements $a_{1}, \ldots, a_{\kappa(k)} \in A$ and $i=1,2, \ldots, \kappa(k)$, $k^{\mathbf{A}}\left(a_{1}, \ldots, a_{\kappa(k)}\right) \neq a_{i}$.

Let $\mathbf{D}$ be a dihypergraph. For each finite subset $V \subseteq V^{\mathbf{D}}$, let

$$
E_{s}^{\mathbf{D}}(V)=\left\{e \in E^{\mathbf{D}}: I_{1}^{\mathbf{D}}(e)=V\right\} \text { and } s^{\mathbf{D}}=\left|E_{s}^{\mathbf{D}}(V)\right| .
$$

Observe that $E_{s}^{\mathbf{D}}(\emptyset)$ contains all the constants of $\mathbf{D}$.

$\mathbf{D}$ is said to be of (dihypergraph) type $\underline{\tau}$ (see [12]), where $\underline{\tau}=\left(\tau_{0}, \tau_{1}, \ldots\right.$ ) is a sequence of cardinal numbers, if

$$
s^{\mathbf{D}}(V) \leq \tau_{|V|} \text { for any finite set } V \subseteq V^{\mathbf{D}} .
$$

A (dihypergraph) type $\underline{\tau}$ is finite, if each of its elements is a non-negative integer, i.e., $\tau \in \mathbb{N}^{\mathbb{N}}$. A finite type $\tau$ is totally finite, if almost all of its terms are zero, i.e., there is $m \in \mathbb{N}$ such that $\tau_{i}=0$ for $i \geq m$.

We say that a dihypergraph $\mathbf{D}$ of finite type $\underline{\tau}$ is total, if $s^{\mathbf{D}}(V)=\tau_{|V|}$ for any finite $V \subseteq V^{\mathbf{D}}$.

It is shown in [13] that if $\mathbf{A}$ is an algebra of type $\langle K, \kappa\rangle$, then $\mathbf{D}(\mathbf{A})$ is a dihypergraph of type $\mathbf{T}(K, \kappa)=\left(T_{0}(K, \kappa), T_{1}(K, \kappa), T_{2}(K, \kappa), \ldots\right)$, where

$$
T_{i}(K, \kappa)=\sum_{m \geq i} \operatorname{sur}(m, i) \cdot\left|\kappa^{-1}(m)\right| \quad \text { for any } i \in \mathbb{N} .
$$


Here $\operatorname{sur}(m, i)$ is the number of all the surjections from an $m$-element set onto an $i$-element set.

Note that $T_{0}(K, \kappa)=\left|\kappa^{-1}(0)\right|$, because $\operatorname{sur}(0,0)=1$ and $\operatorname{sur}(m, 0)=0$ for $m \geq 1$. Moreover, if $\langle K, \kappa\rangle$ is a finite algebraic type (i.e., $K$ is a finite set), then $\mathbf{T}(K, \kappa)$ is totally finite.

It is also easy to see that the following fact holds (the assumption that $\langle K, \kappa\rangle$ is finite, is necessary for the implication $\Longleftarrow)$.

Lemma 2.3. A partial algebra $\mathbf{A}$ of finite type $\langle K, \kappa\rangle$ is total if and only if $\mathbf{D}(\mathbf{A})$ is a total dihypergraph of finite type $\mathbf{T}(K, \kappa)$ (i.e., $s^{\mathbf{D}(\mathbf{A})}(V)=T_{|V|}(K, \kappa)$ for each finite set of vertices $V$ ).

We use subhypergraphs to represent weak subalgebras, therefore we slightly modify the concept of subhypergraph from [4].

More precisely, let $\mathbf{D}$ and $\mathbf{G}$ be dihypergraphs. $\mathbf{G}$ is a subdihypergraph of $\mathbf{D}$ (see [12]), if

$$
V^{\mathbf{G}} \subseteq V^{\mathbf{D}}, \quad E^{\mathbf{G}} \subseteq E^{\mathbf{D}} \text { and } I^{\mathbf{G}}(e)=I^{\mathbf{D}}(e) \text { for any } e \in E^{\mathbf{G}} .
$$

Such subdihypergraphs are called here weak to stress their relation to weak subalgebras.

In an analogous way as for partial algebras we obtain (see also [12]) that the set of all the weak subdihypergraphs of a dihypergraph $\mathbf{D}$ with weak subdihypergraph inclusion $\leq_{w}$, forms a complete and algebraic lattice $\mathcal{S}_{w}(\mathbf{D})=\left\langle S_{w}(\mathbf{D}), \leq_{w}\right\rangle$. Note that a full characterization (in lattice language) of this lattice is the same as that of the weak subalgebra lattice (see [12] for details).

To represent (strong) subalgebras we need the concept of strong subdihypergraph from [12]. A weak subdihypergraph $\mathbf{G}$ of a dihypergraph $\mathbf{D}$ is said to be strong, if $\mathbf{G}$ contains all the hyperedges starting from $\mathbf{G}$, i.e., for any hyperedge $e \in E^{\mathbf{D}}$, if $I_{1}^{\mathbf{D}}(e) \subseteq V^{\mathbf{G}}$, then $e \in E^{\mathbf{G}}$.

It can be shown in the same way as for total algebras (see [12] for details) that the set of all the strong subdihypergraphs of a dihypergraph $\mathbf{D}$ with strong subdihypergraph inclusion $\leq_{s}$ forms a complete and algebraic lattice $\mathcal{S}_{s}(\mathbf{D})=\left\langle S_{s}(\mathbf{D}), \leq_{s}\right\rangle$.

In particular, we have that for any subset $W \subseteq V^{\mathbf{D}}$ there is the least strong subdihypergraph of $\mathbf{D}$ containing $W$, which will be denoted by $\langle W\rangle_{\mathbf{D}}$.

Thus similarly as for algebras, we can say that a dihypergraph $\mathbf{D}$ is locally finite, if for any finite set of vertices $W \subseteq V^{\mathbf{D}},\langle W\rangle_{\mathbf{D}}$ is also finite (i.e., has finitely many vertices).

It is easy to see that if $\mathbf{B}$ is a weak (respectively, strong) subalgebra of a partial algebra $\mathbf{A}$, then its dihypergraph $\mathbf{D}(\mathbf{B})$ is a weak (respectively, strong) subdihypergraph of $\mathbf{D}(\mathbf{A})$. Further,

Theorem 2.4. Let $\mathbf{A}$ be a partial algebra. Then

$$
\mathcal{S}_{w}(\mathbf{A}) \simeq \mathcal{S}_{w}(\mathbf{D}(\mathbf{A})) \quad \text { and } \quad \mathcal{S}_{s}(\mathbf{A}) \simeq \mathcal{S}_{s}(\mathbf{D}(\mathbf{A})) .
$$


This result is obtained in [12] by a direct verification that the function assigning to each strong (respectively weak) subalgebra $\mathbf{B}$ of $\mathbf{A}$ its dihypergraph $\mathbf{D}(\mathbf{B})$, is an isomorphism of lattices. As a simple consequence of this proof, it is also obtained in [12] that for each set $B$ of elements of $\mathbf{A}$,

$$
\mathbf{D}\left(\langle B\rangle_{\mathbf{A}}\right)=\langle B\rangle_{\mathbf{D}(\mathbf{A})}
$$

(where $\langle B\rangle_{\mathbf{A}}$ is the least strong subalgebra of $\mathbf{A}$ containing $B$ ). Thus

Corollary 2.5. A partial algebra $\mathbf{A}$ is locally finite if and only if $\mathbf{D}(\mathbf{A})$ is locally finite.

The following result fundamental for our investigation, is also proved in [12] (its proof is more complicated than the proofs of the previous results)

Theorem 2.6. For arbitrary partial algebras $\mathbf{A}$ and $\mathbf{B}$ (which can even be of different types),

$$
\mathcal{S}_{w}(\mathbf{A}) \simeq \mathcal{S}_{w}(\mathbf{B}) \quad \text { iff } \mathbf{D}(\mathbf{A})^{*} \simeq \mathbf{D}(\mathbf{B})^{*}
$$

\section{Main results}

Results from the previous section reduce our algebraic problem to a hypergraph one. Algebras $\mathbf{A}$ and $\mathbf{B}$ of finite type can be replaced by dihypergraphs $\mathbf{D}$ and $\mathbf{H}$ of totally finite (dihypergraph) type. The other algebraic assumptions are translated in the following way: $\mathbf{D}$ is a total and locally finite dihypergraph without hyperloops, and hypergraphs $\mathbf{D}^{*}$ and $\mathbf{H}^{*}$ are isomorphic. We will prove that then the strong subdihypergraph lattices $\mathcal{S}_{s}(\mathbf{D})$ and $\mathcal{S}_{s}(\mathbf{H})$ are isomorphic, and $\mathbf{H}$ is also total and locally finite without hyperloops.

For a digraph we can invert the orientation of some edges to obtain a new digraph. But the orientation of directed hyperedges can be changed in more than one way, in general. Therefore we need here the following analogous construction for dihypergraphs.

Let $\mathbf{D}$ be a dihypergraph. Let $F \subseteq E_{\text {reg }}^{\mathbf{D}} \backslash E^{\mathbf{D}}(0)$ and $U=\left\{u_{f} \in V^{\mathbf{D}}: f \in\right.$ $F\} \subseteq V^{\mathbf{D}}$ be sets such that

$$
u_{f} \in I_{1}^{\mathbf{D}}(f) \text { for each } f \in F
$$

Then $\mathbf{D}(F, U)$ is the dihypergraph obtained from $\mathbf{D}$ by inverting the orientation of all the hyperedges from $F$ "according" to $U$, i.e.,

$$
V^{\mathbf{D}(F, U)}=V^{\mathbf{D}}, \quad E^{\mathbf{D}(F, U)}=E^{\mathbf{D}}, I^{\mathbf{D}(F, U)}(e)=I^{\mathbf{D}}(e) \text { for any } e \in E^{\mathbf{D}} \backslash F
$$

and

$$
I_{1}^{\mathbf{D}(F, U)}(f)=\left(I_{1}^{\mathbf{D}}(f) \backslash\left\{u_{f}\right\}\right) \cup\left\{I_{2}^{\mathbf{D}}(f)\right\}, \quad I_{2}^{\mathbf{D}(F, U)}(f)=u_{f} \quad \text { for each } f \in F .
$$


Clearly,

$$
\mathbf{D}(F, U)^{*}=\mathbf{D}^{*} .
$$

For dihypergraphs without hyperloops the inverse result also holds.

Lemma 3.1. Let $\mathbf{D}$ and $\mathbf{H}$ be dihypergraphs without hyperloops such that $\mathbf{D}^{*} \simeq \mathbf{H}^{*}$. Then $\mathbf{H} \simeq \mathbf{D}(F, U)$ for some sets $F \subseteq E_{\text {reg }}^{\mathbf{D}} \backslash E^{\mathbf{D}}(0)$ and $U=\left\{u_{f}: f \in F\right\} \subseteq V^{\mathbf{D}}$ such that $u_{f} \in I_{1}^{\mathrm{D}}(f)$ for each $f \in F$.

Proof. Each undirected hyperedge of $\mathbf{D}^{*}$ and of $\mathbf{H}^{*}$ with exactly $k+1$ vertices is the image of a $k$-edge of $\mathbf{D}$ or of $\mathbf{H}$ respectively, under *, because $\mathbf{D}$ and $\mathbf{H}$ do not have hyperloops. Let $\varphi$ be an isomorphism of $\mathbf{D}^{*}$ and $\mathbf{H}^{*}$. Take $F=\left\{e \in E^{\mathbf{D}}\right.$ : $\left.\varphi\left(I_{2}^{\mathrm{D}}(e)\right) \neq I_{2}^{\mathbf{H}}(\varphi(e))\right\}, u_{f}=\varphi^{-1}\left(I_{2}^{\mathbf{H}}(\varphi(f))\right)$ for any $f \in F$ and $U=\left\{u_{f}: f \in F\right\}$. $F$ does not contain 0-edges, because $I^{\mathbf{D}^{*}}(e)=\left\{I_{2}^{\mathrm{D}}(e)\right\}$ for a 0 -edge $e$. Next, $u_{f} \in I_{1}^{\mathrm{D}}(f)$ for $f \in F$ and $\varphi\left(I_{2}^{\mathrm{D}}(e)\right)=I_{2}^{\mathrm{H}}(\varphi(e))$ for any hyperedge $e$ outside $F$. By these facts it easily follows that $\varphi$ is an isomorphism of dihypergraphs $\mathbf{D}(F, U)$ and H.

This lemma is not true for dihypergraphs with hyperloops. For instance, take two dihypergraphs with three vertices and one hyperedge which is a regular 2-edge in the first and a 3 -loop in the second.

Let $\mathbf{D}$ be a dihypergraph, $k \in \mathbb{N} \backslash\{0\}$ and $\mathbf{r}=\left(e_{1}, \ldots, e_{m}\right)$ be a sequence of $k$-edges of $\mathbf{D}$. We say that $\mathbf{r}$ is a $k$-chain, if for each $1 \leq i \leq m-1$,

$$
I_{2}^{\mathrm{D}}\left(e_{i}\right) \in I_{1}^{\mathrm{D}}\left(e_{i+1}\right) \subseteq I_{1}^{\mathrm{D}}\left(e_{i}\right) \cup\left\{I_{2}^{\mathrm{D}}\left(e_{i}\right)\right\} .
$$

If additionally

$$
I_{2}^{\mathrm{D}}\left(e_{m}\right) \in I_{1}^{\mathrm{D}}\left(e_{1}\right) \subseteq I_{1}^{\mathrm{D}}\left(e_{m}\right) \cup\left\{I_{2}^{\mathrm{D}}\left(e_{m}\right)\right\},
$$

then $\mathbf{r}$ is said to be a $k$-cycle.

We denote the set of all the hyperedges of $\mathbf{r}$ by $E^{\mathbf{r}}$.

A $k$-chain (a $k$-cycle) $\mathbf{r}=\left(e_{1}, \ldots, e_{m}\right)$ is a $k$-path (a simple $k$-cycle), if $e_{1}, \ldots, e_{m}$ are pairwise different regular $k$-edges.

We say that a $k$-chain $\mathbf{r}=\left(e_{1}, \ldots, e_{m}\right)$ starts from a set $W \subseteq V^{\mathbf{D}}$ (where $W$ has at least $k$ vertices), if $I_{1}^{\mathrm{D}}\left(e_{1}\right) \subseteq W$. Similarly, $\mathbf{r}$ ends in a vertex $v$, if $I_{2}^{\mathrm{D}}\left(e_{m}\right)=v$.

We say that a sequence of hyperedges is a hyperchain (respectively, a hyperpath, a hypercycle), if it is a $k$-chain (respectively, a $k$-path, a $k$-cycle) for some $k \in$ $\mathbb{N} \backslash\{0\}$. Of course for $k=1$ we obtain ordinary directed chains, paths and cycles as in graph theory.

Definition 3.2. Let $\mathbf{D}$ be a dihypergraph and $W \subseteq V^{\mathbf{D}}$ be a set of vertices. Then

(a) $[W]_{\mathbf{D}}^{q s}$ is a weak subdihypergraph of $\mathbf{D}$ consisting of $W$ and of all the hyperedges (and their endpoints) of all the hyperchains $\left(e_{1}, \ldots, e_{m}\right)$ starting from $W$.

(b) A dihypergraph $\mathbf{D}$ is said to be weakly-locally finite, if for any finite subset $U \subseteq V^{\mathbf{D}},[U]_{\mathbf{D}}^{q s}$ is finite (i.e., has finitely many vertices). 
(c) An algebra is weakly-locally finite, if its dihypergraph is weakly-locally finite.

For any vertex $v$ of $[W]_{\mathbf{D}}^{q s}$, if $v \notin W$, then there is a hyperchain $\left(e_{1}, \ldots, e_{i}\right)$ starting from $W$ and ending in $v$. It is sufficient to take the shortest possible hyperchain containing $v$.

$[W]_{\mathbf{D}}^{q s}$ is contained in $\langle W\rangle_{\mathbf{D}}$, so local finiteness forces weakly-local finiteness. For directed graphs, these two concepts are equivalent, because in this case $[W]_{\mathbf{D}}^{q s}=\langle W\rangle_{\mathbf{D}}$. But generally they are not equal, and consequently the weakly-local finiteness is indeed a weaker condition. For example, take $\mathbf{D}$ with $V^{\mathbf{D}}=\left\{a_{0}, b_{0}, a_{1}, b_{1}, \ldots\right\}, E^{\mathbf{D}}=\left\{e_{0}, f_{0}, e_{1}, f_{1}, \ldots\right\}$ and $I^{\mathbf{D}}\left(e_{i}\right)=\left\langle\left\{a_{i}, b_{i}\right\}, a_{i+1}\right\rangle$, $I^{\mathbf{D}}\left(f_{i}\right)=\left\langle\left\{a_{i}, b_{i}\right\}, b_{i+1}\right\rangle$ for each $i \in \mathbb{N}$. Then $\left\langle\left\{a_{0}, b_{0}\right\}\right\rangle_{\mathbf{D}}=\mathbf{D}$, so $\mathbf{D}$ is not locally finite. On the other hand, $\mathbf{D}$ has only one-element hyperchains, so $\mathbf{D}$ is weaklylocally finite.

Let $R$ be a family of pairwise hyperedge-disjoint simple hypercycles of a dihypergraph $\mathbf{D}$ (note that $R$ may contain $k$-cycles for different $k$ ). For a hyperedge $e \in E^{R}=\bigcup_{\mathbf{r} \in R} E^{\mathbf{r}}$ we take exactly one hypercycle $\left(e_{1}, \ldots, e_{m}\right) \in R$ such that $e=e_{i}$ for exactly one $1 \leq i \leq m$. Next, we take a vertex $u(e) \in I_{1}^{\mathrm{D}}\left(e_{i}\right) \backslash I_{1}^{\mathrm{D}}\left(e_{i+1}\right)$ which is uniquely determined, because $\left|I_{1}^{\mathrm{D}}\left(e_{i}\right) \backslash I_{1}^{\mathrm{D}}\left(e_{i+1}\right)\right|=1$. In this way we obtain a new dihypergraph

$$
\mathbf{D}(R)=\mathbf{D}\left(E^{R}, U\right) \text {, where } U=\left\{u(e): e \in E^{R}\right\} \text { and } E^{R}=\bigcup_{\mathbf{r} \in R} E^{\mathbf{r}} .
$$

For any $\left(e_{1}, \ldots, e_{m}\right) \in R$ we have (where $e_{m+1}=e_{1}$ )

$$
I_{1}^{\mathbf{D}(R)}\left(e_{i}\right)=\left(I_{1}^{\mathbf{D}}\left(e_{i}\right) \backslash\left\{u\left(e_{i}\right)\right\}\right) \cup I_{2}^{\mathbf{D}}\left(e_{i}\right)=I_{1}^{\mathbf{D}}\left(e_{i+1}\right) \text { and } I_{2}^{\mathbf{D}(R)}\left(e_{i}\right)=u\left(e_{i}\right) .
$$

So $\left(e_{m}, \ldots, e_{1}\right)$ is a simple hypercycle in $\mathbf{D}(R)$.

Because $I_{1}^{\mathbf{D}(R)}\left(e_{i}\right) \backslash I_{1}^{\mathbf{D}(R)}\left(e_{i-1}\right)=I_{1}^{\mathbf{D}}\left(e_{i+1}\right) \backslash I_{1}^{\mathbf{D}}\left(e_{i}\right)=\left\{I_{2}^{\mathbf{D}}\left(e_{i}\right)\right\}$, it is not difficult to show that

$$
\mathbf{D}(R)(\bar{R})=\mathbf{D}
$$

where $\bar{R}=\left\{\left(e_{m}, \ldots, e_{1}\right):\left(e_{1}, \ldots, e_{m}\right) \in R\right\}$.

Lemma 3.3. Let $\mathbf{D}$ be a dihypergraph and $R$ be a family of pairwise hyperedgedisjoint simple hypercycles of $\mathbf{D}$. Then

(a) $s^{\mathbf{D}(R)}(V)=s^{\mathbf{D}}(V)$ for each finite $V \subseteq V^{\mathbf{D}}$.

(b) $V^{[W]_{\mathbf{D}(R)}^{q s}}=V^{[W]_{\mathbf{D}}^{q s}}$ and $E^{[W]_{\mathbf{D}(R)}^{q s}}=E^{[W]_{\mathbf{D}}^{q s}}$ for each $W \subseteq V^{\mathbf{D}}$.

Proof. (a): Take a finite set $V \subseteq V^{\mathbf{D}}$ and $\mathbf{r}=\left(e_{1}, \ldots, e_{m}\right) \in R$. Then $e_{i+1}$ starts from $V$ in $\mathbf{D}$ iff $e_{i}$ starts from $V$ in $\mathbf{D}(R)$ for $i=0, \ldots, m-1$ (where $e_{0}=e_{m}$ ). Thus, since $e_{1}, \ldots, e_{m}$ are pairwise different,

$$
\left|E_{s}^{\mathbf{D}}(V) \cap E^{\mathbf{r}}\right|=\left|E_{s}^{\mathbf{D}(R)}(V) \cap E^{\mathbf{r}}\right| .
$$

And consequently, since $R$ contains pairwise hyperedge-disjoint hypercycles,

$$
\left|E_{s}^{\mathbf{D}}(V) \cap E^{R}\right|=\left|E_{s}^{\mathbf{D}(R)}(V) \cap E^{R}\right| .
$$


Hence we obtain (a), because $E_{s}^{\mathbf{D}}(V) \backslash E^{R}=E_{s}^{\mathbf{D}(R)}(V) \backslash E^{R}$ by the definition of $\mathbf{D}(R)$.

(b): We first prove that for any $W \subseteq V^{\mathbf{D}}$,

$$
E^{[W]_{\mathrm{D}}^{q s}} \subseteq E^{[W]_{\mathrm{D}(R)}^{q s}} .
$$

It is sufficient to show that for any $k$-chain $\left(f_{1}, \ldots, f_{n}\right)$ of $\mathbf{D}$ starting from $W$,

$$
\left\{f_{1}, \ldots, f_{n}\right\} \subseteq E^{[W]_{\mathrm{D}(R)}^{q s}} .
$$

If $\left\{f_{1}, \ldots, f_{n}\right\} \cap E^{R}=\emptyset$, then $\left(f_{1}, \ldots, f_{n}\right)$ is also a hyperchain of $\mathbf{D}(R)$ starting from $W$, so $f_{1}, \ldots, f_{n}$ belong to $[W]_{\mathbf{D}(R)}^{q s}$.

Thus we can assume $\left\{f_{1}, \ldots, f_{n}\right\} \cap E^{R} \neq \emptyset$. Assume also that exactly one hyperedge, say, $f_{i}$, belongs to $E^{R}$. The proof of the general case (when several hyperedges of $\left(f_{1}, \ldots, f_{n}\right)$ are contained in some hypercycles of $R$, each of these hypercycles may contain more than one hyperedge of $\left.\left(f_{1}, \ldots, f_{n}\right)\right)$ is only more technically complicated.

Take a $k$-cycle $\left(e_{1}, \ldots, e_{m}\right) \in R$ containing $f_{i}$. We can assume $f_{i}=e_{1}$ (by changing the numeration of $e_{1}, \ldots, e_{m}$, if necessary).

Since $I_{1}^{\mathbf{D}(R)}\left(e_{m}\right)=I_{1}^{\mathbf{D}}\left(e_{1}\right)=I_{1}^{\mathbf{D}}\left(f_{i}\right)$, the sequence $\left(f_{1}, \ldots, f_{i-1}, e_{m}, \ldots, e_{1}\right)$ is a hyperchain in $\mathbf{D}(R)$ starting from $W$ (if $i=1$ we just take $\left(e_{m}, \ldots, e_{1}\right)$ ). So

$$
f_{i}=e_{1} \in E^{[W]_{\mathrm{D}(R)}^{q s}} .
$$

Let $A=I_{1}^{\mathrm{D}}\left(f_{i+1}\right)$.

If $I_{1}^{\mathrm{D}}\left(e_{2}\right)=A$, then it is easy to see that $\left(f_{1}, \ldots, f_{i-1}, e_{m}, \ldots, e_{2}, f_{i+1}, \ldots, f_{n}\right)$ is a hyperchain of $\mathbf{D}(R)$ starting from $W$. This is because $I_{1}^{\mathbf{D}(R)}\left(f_{i+1}\right)=I_{1}^{\mathbf{D}}\left(f_{i+1}\right)=$ $I_{1}^{\mathbf{D}}\left(e_{2}\right)=I_{1}^{\mathbf{D}(R)}\left(e_{1}\right)$ and $\left(e_{m}, \ldots, e_{2}, e_{1}\right)$ is a hypercycle in $\mathbf{D}(R)$.

If $I_{1}^{\mathrm{D}}\left(e_{2}\right) \neq A$ (this is possible for $k$-chains with $k \geq 2$ ), then

$$
A \subseteq I^{\mathbf{D}^{*}}\left(f_{i}\right)=I^{\mathbf{D}^{*}}\left(e_{1}\right)=I^{\mathbf{D}(R)^{*}}\left(e_{1}\right)=I_{1}^{\mathbf{D}(R)}\left(e_{1}\right) \cup\left\{I_{2}^{\mathbf{D}(R)}\left(e_{1}\right)\right\} .
$$

Hence, because $I_{1}^{\mathbf{D}(R)}\left(e_{1}\right)=I_{1}^{\mathbf{D}}\left(e_{2}\right) \neq A$, we have also

$$
I_{2}^{\mathbf{D}(R)}\left(e_{1}\right) \in A \text {. }
$$

Thus in this case $\left(f_{1}, \ldots, f_{i-1}, e_{m}, \ldots, e_{1}, f_{i+1}, \ldots, f_{n}\right)$ is a hyperchain of $\mathbf{D}(R)$ starting from $W$.

Now, since $\mathbf{D}(R)(\bar{R})=\mathbf{D}$, where $\bar{R}=\left\{\left(e_{m}, \ldots, e_{1}\right):\left(e_{1}, \ldots, e_{m}\right) \in R\right\}$, we can apply the previously proved inclusion to dihypergraphs $\mathbf{D}(R)$ and $\mathbf{D}(R)(\bar{R})$ to obtain the inverse inclusion

$$
E^{[W]_{\mathbf{D}(R)}^{q s}} \subseteq E^{[W]_{\mathbf{D}(R)(\bar{R})}^{q s}}=E^{[W]_{\mathrm{D}}^{q s}}
$$

which completes the proof of (b) (because the first equality follows from the second). 
In the proof of the next result we will use the following technical definition. Let $f_{1}, \ldots, f_{m}$ be regular $k$-edges of a dihypergraph $\mathbf{D}$ (where $k \in \mathbb{N} \backslash\{0\}$ ) and let $v_{1}, \ldots, v_{m}$ be vertices of $\mathbf{D}$ such that

$$
v_{i} \in I_{1}^{\mathbf{D}}\left(f_{i}\right) \text { for all } i=1, \ldots, m \text {. }
$$

First, we say that a sequence $\mathbf{r}=\left(\left\langle f_{1}, v_{1}\right\rangle, \ldots,\left\langle f_{m}, v_{m}\right\rangle\right)$ forms a $k$-chain, if for each $i=1, \ldots, m-1$

$$
\left(I_{1}^{\mathbf{D}}\left(f_{i}\right) \backslash\left\{v_{i}\right\}\right) \cup\left\{I_{2}^{\mathbf{D}}\left(f_{i}\right)\right\}=I_{1}^{\mathbf{D}}\left(f_{i+1}\right) .
$$

Secondly, $\mathbf{r}$ forms a $k$-path, if $\mathbf{r}$ forms a $k$-chain and $f_{1}, \ldots, f_{m}$ are pairwise different. Thirdly, $\mathbf{r}$ forms a simple $k$-cycle, if $\mathbf{r}$ forms a $k$-path and $\left(I_{1}^{\mathbf{D}}\left(f_{m}\right) \backslash\right.$ $\left.\left\{v_{m}\right\}\right) \cup\left\{I_{2}^{\mathbf{D}}\left(f_{m}\right)\right\}=I_{1}^{\mathbf{D}}\left(f_{1}\right)$.

If $\mathbf{r}=\left(\left\langle f_{1}, v_{1}\right\rangle, \ldots,\left\langle f_{m}, v_{m}\right\rangle\right)$ forms a $k$-chain, then $\left(f_{1}, \ldots, f_{m}\right)$ is a $k$-chain. But it is easy to see that for $k \geq 2$, the inverse implication is not true. Similarly, $\mathbf{r}$ may form a $k$-path and $\left(f_{1}, \ldots, f_{m}\right)$ may be a simple $k$-cycle, but $\mathbf{r}$ does not form a simple $k$-cycle.

We say (see also [13]) that a dihypergraph $\mathbf{D}$ is a $k$-dihypergraph (where $k \in \mathbb{N}$ ), if all hyperedges of $\mathbf{D}$ are $k$-edges, i.e., $\left|I_{1}^{\mathbf{D}}(e)\right|=k$ for each $e \in E^{\mathbf{D}}$.

A $k$-dihypergraph $\mathbf{D}$ is of $k$-type $\eta$ (where $\eta$ is a cardinal number), if

$$
s^{\mathbf{D}}(V) \leq \eta \text { for each } k \text {-element set } V \subseteq V^{\mathbf{D}} .
$$

$\mathbf{D}$ is of finite $k$-type $n$, if $n$ is a non-negative integer.

We say that a $k$-dihypergraph $\mathbf{D}$ of finite $k$-type $n$ is total, if $s^{\mathbf{D}}(V)=n$ for each $k$-element set $V \subseteq V^{\mathbf{D}}$.

Lemma 3.4. Let a $k$-dihypergraph $\mathbf{D}$ (with $k \in \mathbb{N} \backslash\{0\})$ and sets $F \subseteq E_{\text {reg }}^{\mathbf{D}}$ and $U=\{u(f): f \in F\}$, where $u(f) \in I_{1}^{\mathbf{D}}(f)$ for each $f \in F$, satisfy the following conditions:

(*) $\mathbf{D}$ is a total $k$-dihypergraph of finite $k$-type $n$.

(**) $\mathbf{D}$ is weakly-locally finite.

$(* * *) \mathbf{D}(F, U)$ is a $k$-dihypergraph of finite $k$-type $n$.

Then there is a family $R$ of pairwise hyperedge-disjoint simple $k$-cycles of $\mathbf{D}$ such that

$$
\mathbf{D}(F, U)=\mathbf{D}(R) \text {. }
$$

Proof. Let $\mathbf{H}=\mathbf{D}(F, U)$ and assume $F \neq \emptyset$. It is sufficient to prove that the set $\{\langle f, u(f)\rangle: f \in F\}$ can be divided into sequences $\left\{\left(\left\langle f_{1}^{i}, u\left(f_{1}^{i}\right)\right\rangle, \ldots,\left\langle f_{m_{i}}^{i}, u\left(f_{m_{i}}^{i}\right)\right\rangle\right)\right\}_{i \in I}$ which are pairwise hyperedge-disjoint and each forms a simple $k$-cycle.

Take a $k$-element set $V=\left\{v_{1}, \ldots, v_{k}\right\} \subseteq V^{\mathbf{D}}$ and observe that

$$
E_{s}^{\mathbf{H}}(V)=\left(E_{s}^{\mathbf{D}}(V) \backslash\left\{e \in F: I_{1}^{\mathbf{D}}(e)=V\right\}\right) \cup\left\{e \in F:\left(I_{1}^{\mathbf{D}}(e) \backslash\{u(e)\}\right) \cup\left\{I_{2}^{\mathbf{D}}(e)\right\}=V\right\} .
$$


Hence,

$$
\begin{aligned}
& \left|E_{s}^{\mathbf{D}}(V)\right|-\left|\left\{e \in F: I_{1}^{\mathbf{D}}(e)=V\right\}\right|+\left|\left\{e \in F:\left(I_{1}^{\mathrm{D}}(e) \backslash\{u(e)\}\right) \cup\left\{I_{2}^{\mathrm{D}}(e)\right\}=V\right\}\right| \\
& =\left|E_{s}^{\mathbf{H}}(V)\right| \\
& \leq n=\left|E_{s}^{\mathbf{D}}(V)\right|
\end{aligned}
$$

because $\mathbf{D}$ and $\mathbf{H}$ are of finite $k$-type $n$ and $\mathbf{D}$ is total.

Thus for each $k$-element set $V \subseteq V^{\mathrm{D}}$,

$$
\left|\left\{e \in F:\left(I_{1}^{\mathrm{D}}(e) \backslash\{u(e)\}\right) \cup\left\{I_{2}^{\mathrm{D}}(e)\right\}=V\right\}\right| \leq\left|\left\{e \in F: I_{1}^{\mathrm{D}}(e)=V\right\}\right| .
$$

For any $h \in F$, let $\mathbf{K}_{h}$ be the subdihypergraph of $\mathbf{D}$ containing all the sequences $\left(f_{1}, \ldots, f_{m}\right)$ such that $\left\{f_{1}, \ldots, f_{m}\right\} \subseteq F, I_{1}^{\mathrm{D}}\left(f_{1}\right)=I_{1}^{\mathrm{D}}(h)$ and $\left(\left\langle f_{1}, u\left(f_{1}\right)\right\rangle, \ldots,\left\langle f_{m}, u\left(f_{m}\right)\right\rangle\right)$ forms a $k$-chain.

Then $h \in E^{\mathbf{K}_{h}}$, and $\mathbf{K}_{h}$ is a weak subdihypergraph of $[W]_{\mathbf{D}}^{q s}$. By $(* *),[W]_{\mathbf{D}}^{q s}$ is finite, so $\mathbf{K}_{h}$ is also finite. Hence $\mathbf{K}_{h}$ has only finitely many hyperedges, because $\mathbf{K}_{h}$ (being a subdihypergraph of $\mathbf{D}$ ) is of finite $k$-type $n$.

Let $f \in E^{\mathbf{K}_{h}}$ and $V_{f}=\left(I_{1}^{\mathbf{D}}(f) \backslash\{u(f)\}\right) \cup\left\{I_{2}^{\mathbf{D}}(f)\right\}$. Then

$$
E_{s}^{\mathbf{K}_{h}}\left(V_{f}\right)=\left\{e \in F: I_{1}^{\mathbf{D}}(e)=V_{f}\right\} .
$$

The inclusion $\subseteq$ is obvious. Take $e \in F$ such that $I_{1}^{\mathrm{D}}(e)=V_{f}$. Since $f \in$ $E^{\mathbf{K}_{h}}$, there is a sequence $\left(\left\langle f_{1}, u\left(f_{1}\right)\right\rangle, \ldots,\left\langle f_{m}, u\left(f_{m}\right)\right\rangle\right)$ forming a $k$-chain such that $f_{1}, \ldots, f_{m} \in F, I_{1}^{\mathrm{D}}\left(f_{1}\right)=I_{1}^{\mathrm{D}}(h)$ and $f=f_{m}$. By the definition of $V_{f}$, $\left(\left\langle f_{1}, u\left(f_{1}\right)\right\rangle, \ldots,\left\langle f_{m}, u\left(f_{m}\right)\right\rangle,\langle e, u(e)\rangle\right)$ also forms such a $k$-chain. Hence $e \in E^{\mathbf{K}_{h}}$, so $e \in E_{s}^{\mathbf{K}_{h}}\left(V_{f}\right)$.

Since $E^{\mathbf{K}_{h}} \subseteq F$, the above equality and (1) imply that for any $f \in E^{\mathbf{K}_{h}}$,

$$
\left|\left\{e \in E^{\mathbf{K}_{h}}:\left(I_{1}^{\mathbf{D}}(e) \backslash\{u(e)\}\right) \cup\left\{I_{2}^{\mathbf{D}}(e)\right\}=V_{f}\right\}\right| \leq\left|E_{s}^{\mathbf{K}_{h}}\left(V_{f}\right)\right| .
$$

Now we prove that there is a sequence $\left(\left\langle f_{1}, u\left(f_{1}\right)\right\rangle, \ldots,\left\langle f_{p}, u\left(f_{p}\right)\right\rangle\right)$ forming a simple $k$-cycle and containing all the $k$-edges of $\mathbf{K}_{h}$.

The family $\mathcal{S}$ of all sequences which form $k$-paths in $\mathbf{K}_{h}$ starting from $I_{1}^{\mathrm{D}}(h)$ is non-empty (because it contains the one-element sequence $\langle h, u(h)\rangle)$ and finite (because $\mathbf{K}_{h}$ has only finitely many $k$-edges). Thus we can take $\mathbf{r}=$ $\left(\left\langle f_{1}, u\left(f_{1}\right)\right\rangle, \ldots,\left\langle f_{p}, u\left(f_{p}\right)\right\rangle\right) \in \mathcal{S}$ with maximal length.

First, $\mathbf{r}$ is a simple $k$-cycle. Assume otherwise that $I_{1}^{\mathbf{D}}\left(f_{1}\right) \neq W$, where $W=$ $\left(I_{1}^{\mathbf{D}}\left(f_{p}\right) \backslash\left\{u\left(f_{p}\right)\right\}\right) \cup\left\{I_{2}^{\mathbf{D}}\left(f_{p}\right)\right\}$. Observe that $f_{i}$ (for $i \leq p-1$ ) ends in $W$ (i.e., $\left.I_{1}^{\mathrm{D}}\left(f_{i}\right) \backslash\left\{u\left(f_{i}\right)\right\} \cup I_{2}^{\mathrm{D}}\left(f_{i}\right)=W\right)$ if and only if $f_{i+1}$ starts from $W$ (i.e., $I_{1}^{\mathrm{D}}\left(f_{i+1}\right)=W$ ). Hence, and by the assumption, since $f_{1}, \ldots, f_{p}$ are pairwise different, we obtain that the number of all the $k$-edges of $\mathbf{r}$ ending in $W$ is greater than the number of all the $k$-edges of $\mathbf{r}$ starting from $W$. Thus by (2) there is a $k$-edge $f$ starting from $W$ that does not belong to $\mathbf{r}$. But then $\left(\left\langle f_{1}, u\left(f_{1}\right)\right\rangle, \ldots,\left\langle f_{p}, u\left(f_{p}\right)\right\rangle,\langle f, u(f)\rangle\right)$ forms a $k$-path in $\mathbf{K}_{h}$ starting from $I_{1}^{\mathrm{D}}(h)$ and its length is $p+1$, which is impossible. 
Secondly,

$$
\left\{f_{1}, \ldots, f_{p}\right\}=E^{\mathbf{K}_{h}} .
$$

Assume otherwise that there is a $k$-edge $f$ of $E^{\mathbf{K}_{h}}$ such that $f \notin\left\{f_{1}, \ldots, f_{p}\right\}$. Then by the definition of $\mathbf{K}_{h}$, there is a sequence $\left(\left\langle e_{1}, u\left(e_{1}\right)\right\rangle, \ldots,\left\langle e_{m}, u\left(e_{m}\right)\right\rangle\right)$ forming a $k$-chain such that $e_{m}=f$ and $I_{1}^{\mathrm{D}}\left(e_{1}\right)=I_{1}^{\mathrm{D}}(h)=I_{1}^{\mathrm{D}}\left(f_{1}\right)$. Take the first $k$-edge of $\left(e_{1}, \ldots, e_{m}\right)$, say, $g=e_{j}$, which does not belong to $\mathbf{r}$. Next, let $f_{i_{0}}$ be a regular $k$-edge of $\mathbf{r}$ such that

$$
f_{i_{0}}= \begin{cases}e_{j-1} & \text { if } j \geq 2 \\ f_{p} & \text { if } j=1\end{cases}
$$

Then we have

$$
I_{2}^{\mathrm{D}}\left(f_{i_{0}}\right) \in I_{1}^{\mathrm{D}}(g) \subseteq I_{1}^{\mathrm{D}}\left(f_{i_{0}}\right) \cup\left\{I_{2}^{\mathrm{D}}\left(f_{i_{0}}\right)\right\}
$$

(if $j=1$, then $I_{1}^{\mathrm{D}}(g)=I_{1}^{\mathrm{D}}(h)=I_{1}^{\mathrm{D}}\left(f_{1}\right)$, so we use the fact that $\mathbf{r}$ is a simple $k$-cycle).

Observe that $\mathbf{K}_{g}$ is contained in $\mathbf{K}_{h}$. If $j=1$ (i.e., $\left.g=e_{1}\right)$, then $I_{1}^{\mathrm{D}}(g)=I_{1}^{\mathrm{D}}(h)$, so $\mathbf{K}_{g}=\mathbf{K}_{h}$. If $j \geq 2$, then for each $\left(\left\langle a_{1}, u\left(a_{1}\right)\right\rangle, \ldots,\left\langle a_{l}, u\left(a_{l}\right)\right\rangle\right)$ starting from $I_{1}^{\mathrm{D}}(g)$ we have (by (3)) that $\left(\left\langle f_{1}, u\left(f_{1}\right)\right\rangle, \ldots,\left\langle f_{i_{0}}, u\left(f_{i_{0}}\right)\right\rangle,\left\langle a_{1}, u\left(a_{1}\right)\right\rangle, \ldots,\left\langle a_{l}, u\left(a_{l}\right)\right\rangle\right)$ forms a $k$-chain starting from $I_{1}^{\mathbf{D}}(h)$.

Let $\overline{\mathbf{K}}$ be a $k$-dihypergraph consisting of all the sequences $\left(e_{1}, \ldots, e_{m}\right)$ such that $I_{1}^{\mathrm{D}}\left(e_{1}\right)=I_{1}^{\mathrm{D}}(g),\left\{e_{1}, \ldots, e_{m}\right\} \subseteq F \backslash\left\{f_{1}, \ldots, f_{p}\right\}$ and $\left(\left\langle e_{1}, u\left(e_{1}\right)\right\rangle, \ldots,\left\langle e_{m}, u\left(e_{m}\right)\right\rangle\right)$ forms a $k$-chain. Obviously $g \in E^{\overline{\mathrm{K}}}$.

Take a $k$-element set $V \subseteq V^{\mathbf{D}}$. Since $\mathbf{r}$ forms a simple $k$-cycle, we deduce that the number of all the $k$-edges of $\mathbf{r}$ starting from $V$ equals the number of all the $k$-edges of $\mathbf{r}$ ending in $V$. Hence

$$
\begin{aligned}
& \left|\left\{e \in F:\left(I_{1}^{\mathbf{D}}(e) \backslash\{u(e)\}\right) \cup\left\{I_{2}^{\mathbf{D}}(e)\right\}=V\right\} \cap\left\{f_{1}, \ldots, f_{p}\right\}\right| \\
& =\left|\left\{e \in F: I_{1}^{\mathbf{D}}(e)=V\right\} \cap\left\{f_{1}, \ldots, f_{p}\right\}\right| .
\end{aligned}
$$

Thus by (1),

$\left|\left\{e \in F:\left(I_{1}^{\mathbf{D}}(e) \backslash\{u(e)\}\right) \cup\left\{I_{2}^{\mathbf{D}}(e)\right\}=V\right\} \backslash\left\{f_{1}, \ldots, f_{p}\right\}\right| \leq\left|\left\{e \in F: I_{1}^{\mathbf{D}}(e)=V\right\} \backslash\left\{f_{1}, \ldots, f_{p}\right\}\right|$.

In exactly the same way as for $\mathbf{K}_{h}$ we can prove that for each $f \in E^{\overline{\mathbf{K}}}$,

$$
E_{s}^{\overline{\mathbf{K}}}\left(V_{f}\right)=\left\{e \in F: I_{1}^{\mathrm{D}}(e)=V_{f}\right\} \backslash\left\{f_{1}, \ldots, f_{p}\right\},
$$

where $V_{f}=\left(I_{1}^{\mathbf{D}}(f) \backslash\{u(f)\}\right) \cup\left\{I_{2}^{\mathbf{D}}(f)\right\}$.

Since $E^{\overline{\mathbf{K}}} \subseteq F \backslash\left\{f_{1}, \ldots, f_{p}\right\}$, the above two facts imply

$$
\left|\left\{e \in E^{\overline{\mathbf{K}}}:\left(I_{1}^{\mathbf{D}}(e) \backslash\{u(e)\}\right) \cup\left\{I_{2}^{\mathbf{D}}(e)\right\}=V_{f}\right\}\right| \leq\left|E_{s}^{\overline{\mathbf{K}}}\left(V_{f}\right)\right| .
$$

Using this inequality it can be shown, in the same way as for $\mathbf{K}_{h}$, that there is a sequence $\left.\left\langle\bar{f}_{1}, u\left(\bar{f}_{1}\right)\right\rangle, \ldots,\left\langle\bar{f}_{q}, u\left(\bar{f}_{q}\right)\right\rangle\right)$ which forms a simple $k$-cycle in $\overline{\mathbf{K}}$ starting 
from $I_{1}^{\mathrm{D}}(g)$. Then by $(3),\left(\left\langle f_{1}, u\left(f_{1}\right)\right\rangle, \ldots,\left\langle f_{i_{0}}, u\left(f_{i_{0}}\right)\right\rangle,\left\langle\bar{f}_{1}, u\left(\bar{f}_{1}\right)\right\rangle, \ldots,\left\langle\bar{f}_{q}, u\left(\bar{f}_{q}\right)\right\rangle\right.$, $\left.\left\langle f_{i_{0}+1}, u\left(f_{i_{0}+1}\right)\right\rangle, \ldots,\left\langle f_{p}, u\left(f_{p}\right)\right\rangle\right)$ forms a simple $k$-cycle starting from $I_{1}^{\mathrm{D}}(h)$ and its length is $q+p>p$. But this is impossible, so $\mathbf{r}$ contains all the $k$-edges of $\mathbf{K}_{h}$.

Take now two $k$-edges $e, f \in F$ and their dihypergraphs $\mathbf{K}_{e}, \mathbf{K}_{f}$. Let $\left(\left\langle e_{1}, u\left(e_{1}\right)\right\rangle, \ldots,\left\langle e_{m}, u\left(e_{m}\right)\right\rangle\right)$ and $\left(\left\langle f_{1}, u\left(f_{1}\right)\right\rangle, \ldots,\left\langle f_{l}, u\left(f_{l}\right)\right\rangle\right)$ be simple $k$-cycles that start from $I_{1}^{\mathrm{D}}(e)$ and $I_{1}^{\mathrm{D}}(f)$, respectively, and contain all the $k$-edges of $\mathbf{K}_{e}$ and $\mathbf{K}_{f}$.

Assume that $e_{i}=f_{j}$ for some $1 \leq i \leq m, 1 \leq j \leq l$. Then $\left(\left\langle e_{1}, u\left(e_{1}\right)\right\rangle\right.$, $\left.\ldots,\left\langle e_{i}, u\left(e_{i}\right)\right\rangle,\left\langle f_{j+1}, u\left(f_{j+1}\right)\right\rangle, \ldots,\left\langle f_{l}, u\left(f_{l}\right)\right\rangle,\left\langle f_{1}, u\left(f_{1}\right)\right\rangle, \ldots,\left\langle f_{j}, u\left(f_{j}\right)\right\rangle\right)$ forms a $k$-chain starting from $I_{1}^{\mathrm{D}}(e)$ (if $j=l$, then we take $f_{j+1}=f_{1}$ ). Hence $E^{\mathbf{K}_{f}}=$ $\left\{f_{1}, \ldots, f_{l}\right\} \subseteq E^{\mathbf{K}_{e}}$. Analogously $\left\{e_{1}, \ldots, e_{m}\right\} \subseteq E^{\mathbf{K}_{f}}$, so $E^{\mathbf{K}_{e}}=E^{\mathbf{K}_{f}}$.

Thus we have shown that for any $e, f \in F$ the dihypergraphs $\mathbf{K}_{e}$ and $\mathbf{K}_{f}$ are hyperedge-disjoint or have the same sets of hyperedges (i.e., they are equal). Now to end the proof it is sufficient to take all the pairwise different dihypergraphs of the form $\mathbf{K}_{f}$, where $f \in F$. Next, for each of these dihypergraphs we take $\left(\left\langle f_{1}, u\left(f_{1}\right)\right\rangle, \ldots,\left\langle f_{p}, u\left(f_{p}\right)\right\rangle\right)$ that forms a simple $k$-cycle and contains all its $k$-edges.

Lemma 3.5. Let $\mathbf{D}$ be a total weakly-locally finite dihypergraph of totally finite type $\underline{\tau}$ without hyperloops. Let $\mathbf{H}$ be a dihypergraph of type $\underline{\tau}$ such that $\mathbf{D}^{*} \simeq \mathbf{H}^{*}$. Then $\mathbf{H}$ has no hyperloops.

Proof. Assume that $\tau$ is a non-zero sequence. Otherwise $\mathbf{D}$ and $\mathbf{H}$ are dihypergraphs without hyperedges. Thus since $\underline{\tau}$ is a totally finite type, there is $M \in \mathbb{N}$ such that $\tau_{i}=0$ for $i \geq M+1$ and $\tau_{M} \in \bar{N} \backslash\{0\}$. Then $\mathbf{D}$ and $\mathbf{H}$ have no $i$-edges for $i \geq M+1$, in particular $\mathbf{H}$ has no $(M+1)$-loops.

Take weak subdihypergraphs $\mathbf{D}_{1}, \mathbf{H}_{1}$ of $\mathbf{D}$ and $\mathbf{H}$, respectively, consisting of all the vertices and regular $M$-edges. Then $\mathbf{D}_{1}$ and $\mathbf{H}_{1}$ are $M$-dihypergraphs of $M$-type $\tau_{M} . \mathbf{D}_{1}$ is weakly-locally finite. Since $\mathbf{D}$ has no hyperloops, $\mathbf{D}_{1}$ contains all the $M$-edges of $\mathbf{D}$, and in particular, $\mathbf{D}_{1}$ is total.

Since $\mathbf{D}$ and $\mathbf{H}$ have no $(M+1)$-loops, each hyperedge of $\mathbf{D}^{*}$ and of $\mathbf{H}^{*}$ with exactly $M+1$ endpoints is the image of a regular $M$-edge under *. Thus $\mathbf{D}_{1}^{*}$ and $\mathbf{H}_{1}^{*}$ are subhypergraphs of $\mathbf{D}^{*}$ and $\mathbf{H}^{*}$, respectively, containing all the hyperedges with exactly $M+1$ endpoints. Hence we infer $\mathbf{D}_{1}^{*} \simeq \mathbf{H}_{1}^{*}$. Since $\mathbf{D}_{1}, \mathbf{H}_{1}$ are $M$-dihypergraphs without hyperloops, we can apply Lemmas 3.1, 3.4. Thus $\mathbf{H}_{1} \simeq$ $\mathbf{D}_{1}\left(R_{1}\right)$, where $R_{1}$ is a family of pairwise hyperedge-disjoint simple $M$-cycles. This fact and Lemma 3.3 imply $s^{\mathbf{H}_{1}}(V)=s^{\mathbf{D}_{1}}(V)=\tau_{M}$ for each $M$-element $V \subseteq V^{\mathbf{H}}$. Hence we deduce that $\mathbf{H}_{1}$ contains all the $M$-edges of $\mathbf{H}$, because $\mathbf{H}$ is of type $\underline{\tau}$ and $\tau_{M}$ is a natural number. Thus $\mathbf{H}$ has no $M$-loops.

Now, take subdihypergraphs $\mathbf{D}_{2}, \mathbf{H}_{2}$ of $\mathbf{D}$ and $\mathbf{H}$, respectively, consisting of all the regular $(M-1)$-edges. Using the fact that $\mathbf{D}$ and $\mathbf{H}$ have no $M$-loops, we can show in exactly the same way as above that $\mathbf{H}_{2}$ contains all the $(M-1)$-edges of $\mathbf{H}$, and consequently, $\mathbf{H}$ has no $(M-1)-$ loops.

Thus by simple induction we obtain that $\mathbf{H}$ has no hyperloops. 
The above fact is not true for finite types that are not totally finite. For example, take a finite type $(1,1,1, \ldots)$ and let $\mathbf{D}$ be an infinite total dihypergraph without hyperloops of this type. We construct the dihypergraph $\mathbf{H}$ in the following way: Exactly one constant (i.e., $0-$ edge) $e_{0}$ of $\mathbf{D}$ is replaced by a loop $f_{0}$ in this vertex (i.e., $I_{1}^{\mathbf{H}}\left(f_{0}\right)=\left\{I_{2}^{\mathbf{D}}\left(e_{0}\right)\right\}$ and $\left.I_{2}^{\mathbf{H}}\left(f_{0}\right)=I_{2}^{\mathbf{D}}\left(e_{0}\right)\right)$. Exactly one 1 -edge $e_{1}$ of $\mathbf{D}$ starting from $I_{1}^{\mathbf{H}}\left(f_{0}\right)$ is replaced by a 2-loop $f_{1}$ with endpoints of $e_{1}$ (i.e., $I_{1}^{\mathbf{H}}\left(f_{1}\right)=$ $I_{1}^{\mathbf{D}}\left(e_{1}\right) \cup\left\{I_{2}^{\mathbf{D}}\left(e_{1}\right)\right\}$ and $\left.I_{2}^{\mathbf{H}}\left(f_{1}\right)=I_{2}^{\mathbf{D}}\left(e_{1}\right)\right)$. And so on. It is easy to see that $\mathbf{H}$ is also of type $(1,1,1, \ldots)$ and $\mathbf{H}^{*} \simeq \mathbf{D}^{*}$. Obviously $\mathbf{H}$ is not total (for example, it has no 0 -edges).

Theorem 3.6. Let dihypergraphs $\mathbf{D}$ and $\mathbf{H}$ satisfy the following conditions:

(*) $\mathbf{D}^{*} \simeq \mathbf{H}^{*}$,

(**) $\mathbf{D}$ is a total weakly-locally finite dihypergraph of totally finite type $\underline{\tau}$ without hyperloops,

$(* * *) \mathbf{H}$ is a dihypergraph of totally finite type $\underline{\tau}$.

Then

(a) $\mathcal{S}_{s}(\mathbf{H}) \simeq \mathcal{S}_{s}(\mathbf{D})$.

(b) $\mathbf{H}$ is also a total weakly-locally finite dihypergraph of totally finite type $\underline{\tau}$ without hyperloops.

(c) If $\mathbf{D}$ is locally finite, then $\mathbf{H}$ is also locally finite.

Proof. By Lemma 3.5, $\mathbf{H}$ has no hyperloops either. So by Lemma 3.1, there are $F \subseteq E_{\text {reg }}^{\mathbf{D}} \backslash E^{\mathbf{D}}(0)$ and $U=\{u(f): f \in F\} \subseteq V^{\mathbf{D}}$ such that $u(f) \in I_{1}^{\mathbf{D}}(f)$ for any $f \in F$ and

$$
\mathbf{H} \simeq \mathbf{D}(F, U)
$$

For each $k \in \mathbb{N} \backslash\{0\}$, let $\mathbf{D}_{k}$ be a weak subdihypergraph of $\mathbf{D}$ consisting of all the vertices and $k$-edges of $\mathbf{D}$. Then $\mathbf{D}_{k}$ is a total weakly-locally finite $k$-dihypergraph of $k$-type $\tau_{k}$.

Next, let $F_{k}$ be the set of all the $k$-edges of $F$ and $U_{k}=\left\{u(f): f \in F_{k}\right\}$. Then $F_{k} \subseteq E^{\mathbf{D}_{k}}, U_{k} \subseteq V^{\mathbf{D}_{k}}$ and $F_{k} \cap F_{l}=\emptyset$ for $k \neq l$. Further, the $k$-dihypergraph $\mathbf{D}_{k}\left(F_{k}, U_{k}\right)$ is a weak subdihypergraph of $\mathbf{D}(F, U)$. Thus $\mathbf{D}_{k}\left(F_{k}, U_{k}\right)$ is of $k$-type $\tau_{k}$. Applying Lemma 3.4 we obtain

$$
\mathbf{D}_{k}\left(F_{k}, U_{k}\right)=\mathbf{D}_{k}\left(R_{k}\right),
$$

where $R_{k}$ is a family of pairwise hyperedge-disjoint simple $k$-cycles of $\mathbf{D}_{k}$, and thus also of $\mathbf{D}$.

Let $R=\bigcup_{k \in \mathbb{N} \backslash\{0\}} R_{k} . R_{k}$ and $R_{l}$ are disjoint for $k \neq l$, because sets of $k$-edges and of $l$-edges are disjoint for $k \neq l$. Thus $R$ is a family of pairwise hyperedgedisjoint simple hypercycles of $\mathbf{D}$. And by the above equality we infer

$$
\mathbf{D}(F, U)=\mathbf{D}(R) \text {. }
$$


Hence and by Lemma 3.3, $\mathbf{H}$ is a total weakly-locally finite dihypergraph of totally finite type $\underline{\tau}$.

Take now a strong subdihypergraph $\mathbf{K}$ of $\mathbf{D}$. If $\left(f_{1}, \ldots, f_{m}\right)$ is a hypercycle such that $I_{1}^{\mathbf{D}}\left(f_{i}\right) \subseteq V^{\mathbf{K}}$ for some $1 \leq i \leq m$, then $\left\{f_{1}, \ldots, f_{m}\right\} \subseteq E^{\mathbf{K}}$. As $I_{1}^{\mathbf{D}}\left(f_{i}\right) \subseteq V^{\mathbf{K}}$ implies $I_{2}^{\mathrm{D}}\left(f_{i}\right) \in V^{\mathbf{K}}$, we have $I_{1}^{\mathrm{D}}\left(f_{i+1}\right) \subseteq I_{1}^{\mathrm{D}}\left(f_{i}\right) \cup\left\{I_{2}^{\mathrm{D}}\left(f_{i}\right)\right\} \subseteq V^{\mathbf{K}}$ (where $f_{m+1}=$ $\left.f_{1}\right)$ and so on.

Let $\overline{\mathbf{K}}$ be a weak subdihypergraph of $\mathbf{D}(R)$ consisting of all the vertices and hyperedges of $\mathbf{K}$. Take a hyperedge $e$ starting from $\overline{\mathbf{K}}$, i.e., $I_{1}^{\mathbf{D}(R)}(e) \subseteq V^{\overline{\mathbf{K}}}=V^{\mathbf{K}}$. If $e \notin E^{R}$, then $e$ starts from $V^{\mathbf{K}}$ also in $\mathbf{D}$, so $e \in E^{\mathbf{K}}=E^{\overline{\mathbf{K}}}$. If $e \in E^{R}$, then $e=f_{i}$ for some $\left(f_{1}, \ldots, f_{m}\right) \in R$. Since $I_{1}^{\mathbf{D}}\left(f_{i+1}\right)=I_{1}^{\mathbf{D}(R)}(e)$ (here $f_{m+1}=f_{1}$ ), the whole hypercycle belongs to $\mathbf{K}$, and thus also to $\overline{\mathbf{K}}$. In particular, $e \in E^{\overline{\mathbf{K}}}$. Summarizing, $\overline{\mathbf{K}}$ is a strong subdihypergraph of $\mathbf{D}(R)$.

It is also easy to show (see [12]) that for any strong subdihypergraphs $\mathbf{K}_{1}$ and $\mathbf{K}_{2}$ of any dihypergraph $\mathbf{H}, \mathbf{K}_{1}$ is a strong subdihypergraph of $\mathbf{K}_{2}$ iff $V^{\mathbf{K}_{1}} \subseteq V^{\mathbf{K}_{2}}$. In particular, $\mathbf{K}_{1}=\mathbf{K}_{2}$ iff $V^{\mathbf{K}_{1}}=V^{\mathbf{K}_{2}}$. Thus the function $\varphi: S_{s}(\mathbf{D}) \longrightarrow S_{s}(\mathbf{D}(R))$ such that $\varphi(\mathbf{K})=\overline{\mathbf{K}}$, is injective and preserves the strong subdihypergraph inclusion $\leq_{s}$.

$\bar{R}=\left\{\left(e_{m}, \ldots, e_{1}\right):\left(e_{1}, \ldots, e_{m}\right) \in R\right\}$ is a family of hypercycles of $\mathbf{D}(R)$ and $\mathbf{D}=\mathbf{D}(R)(\bar{R})$. So we can apply the above results (proved for $\mathbf{D}$ and $R)$ to $\mathbf{D}(R)$ and $\bar{R}$. In this way we obtain that the function $\psi: S_{s}(\mathbf{D}(R)) \longrightarrow S_{s}(\mathbf{D})$ defined analogously as $\varphi$, is injective and preserves $\leq_{s}$. Moreover, $\varphi \circ \psi$ and $\psi \circ \varphi$ are identities. This shows that $\varphi$ is an isomorphism of lattices $\mathbf{S}_{s}(\mathbf{D})$ and $\mathbf{S}_{s}(\mathbf{D}(R))$. By the definition of $\varphi$ we also have (c).

Corollary 3.7. Let $\mathbf{A}=\left\langle A,\left(k^{\mathbf{A}}\right)_{k \in K}\right\rangle$ and $\mathbf{B}=\left\langle B,\left(k^{\mathbf{B}}\right)_{k \in K}\right\rangle$ be algebras of finite type $\langle K, \kappa\rangle$ such that

$(*) \mathcal{S}_{w}(\mathbf{B}) \simeq \mathcal{S}_{w}(\mathbf{A})$

(**) $\mathbf{A}$ is a total weakly-locally finite algebra,

$(* * *)$ for each $k \in K, a_{1}, \ldots, a_{\kappa(k)} \in A$ and $1 \leq i \leq \kappa(k), k^{\mathbf{A}}\left(a_{1}, \ldots, a_{\kappa(k)}\right) \neq a_{i}$.

Then

(a) $\mathcal{S}_{s}(\mathbf{B}) \simeq \mathcal{S}_{s}(\mathbf{A})$,

(b) $\mathbf{B}$ is a total weakly-locally finite algebra.

(c) For each $k \in K, b_{1}, \ldots, b_{\kappa(k)} \in B$ and $1 \leq i \leq \kappa(k), k^{\mathbf{B}}\left(b_{1}, \ldots, b_{\kappa(k)}\right) \neq b_{i}$.

Proof. The proof follows directly from Lemmas 2.2, 2.3, Theorems 2.4, 2.6, 3.6 and Corollary 2.5 .

Corollary 3.8. Let $\mathbf{A}=\left\langle A,\left(k^{\mathbf{A}}\right)_{k \in K}\right\rangle$ and $\mathbf{B}=\left\langle B,\left(k^{\mathbf{B}}\right)_{k \in K}\right\rangle$ be algebras of finite type $\langle K, \kappa\rangle$ such that

$(*) \mathcal{S}_{w}(\mathbf{B}) \simeq \mathcal{S}_{w}(\mathbf{A})$, 
(**) A is a locally finite total algebra,

$(* * *)$ for each $k \in K, a_{1}, \ldots, a_{\kappa(k)} \in A$ and $1 \leq i \leq \kappa(k), k^{\mathbf{A}}\left(a_{1}, \ldots, a_{\kappa(k)}\right) \neq a_{i}$.

Then

(a) $\mathcal{S}_{s}(\mathbf{B}) \simeq \mathcal{S}_{s}(\mathbf{A})$,

(b) $\mathbf{B}$ is a locally finite total algebra.

(c) For each $k \in K, b_{1}, \ldots, b_{\kappa(k)} \in B$ and $1 \leq i \leq \kappa(k), k^{\mathbf{B}}\left(b_{1}, \ldots, b_{\kappa(k)}\right) \neq b_{i}$.

A particular case of the above results for unary algebras (and for directed graphs) is proved in [11]. [11] contains also examples which show the necessity of the conditions that a unary algebra be total, locally finite and of finite type. Recall also that weakly-local finiteness and local finiteness are equivalent for unary algebras.

Finally note that the condition $(* * *)$ of Theorem 3.6 is used in the proof (see e.g., Lemma 3.1). We do not know, however, whether it is indeed necessary.

\section{References}

[1] Bartol W., Weak subalgebra lattices, Comment. Math. Univ. Carolinae, 31(1990), 405-410.

[2] Bartol W., Weak subalgebra lattices of monounary partial algebras, Comment. Math. Univ. Carolinae, 31(1990), 411-414.

[3] Bartol W., Rosselló F., Rudak L., Lectures on Algebras, Equations and Partiality, Technical report B-006, Univ. Illes Balears, Dept. Ciencies Mat. Inf., ed. Rosselló F., 1992.

[4] Berge C., Graphs and Hypergraphs, North-Holland, Amsterdam, 1973.

[5] Burmeister P., A Model Theoretic Oriented Approach to Partial Algebras, Math. Research Band, 32, Akademie Verlag, Berlin, 1986.

[6] Crawley P., Dilworth R.P., Algebraic Theory of Lattices, Prentice Hall Inc., Englewood Cliffs, NJ, 1973.

[7] Burris S., Sankappanavar H. P., A Course in Universal Algebra, Graduate Texts in Mathematics, 78. Springer-Verlag, New York-Berlin, 1981.

[8] Evans T., Ganter B., Varieties with modular subalgebra lattices, Bull. Austr. Math. Soc., 28(1983), 247-254.

[9] Jónsson B., Topics in Universal Algebra, Lecture Notes in Mathemathics 250, Springer-Verlag, 1972.

[10] McKenzie R. N., McNulty G. F., Taylor W. F., Algebras, Lattices, Varieties v.I, Wadsworth and Brooks/Cole Advanced Books and Software, Monterey, 1987. 
[11] Pióro K., On a strong property of the weak subalgebra lattice, Alg. Univ., 40(4)(1998), 477-495.

[12] Pióro K., On connections between hypergraphs and algebras, Arch. Math. (Brno), 36(1) (2000), 45-60.

[13] Pióro K., Some properties of the weak subalgebra lattice of a partial algebra of a fixed type, Arch. Math. (Brno), 38(2)(2002), 81-94

[14] Schmidt R., Subgroup Lattices of Groups, Walter de Gruyter, New York, 1994.

[15] Shapiro J., Finite equational bases for subalgebra distributive varieties, Alg. Univ., 24(1987), 36-40.

[16] Shapiro J., Finite algebras with abelian properties, Alg. Univ., 25(1988), 334-364. 